\title{
Fatty acid profile and estimated desaturase activities in whole blood are associated with metabolic health
}

\author{
Karianne Svendsen ${ }^{1,2 \dagger}$, Thomas Olsen ${ }^{1 \dagger}$, Tove C. Nordstrand Rusvik', Stine M. Ulven ${ }^{1}$, Kirsten B. Holven ${ }^{1,3}$,
} Kjetil Retterstø| $\left.\right|^{1,2}$ and Vibeke H. Telle-Hansen ${ }^{4^{*}}$ (D)

\begin{abstract}
Background: The aim was to investigate if fatty acid profile and estimated desaturase activities; stearoyl CoAdesaturase (SCD), delta-5-desaturase and delta-6-desaturase (D5D; D6D), differ between individuals with metabolically healthy $(\mathrm{MH})$ and unhealthy $(\mathrm{MU})$ phenotypes. We also explored these associations according to BMI categories.

Methods: Men and women at moderately elevated risk of cardiovascular disease were included in this crosssectional study $(n=321)$. If subjects met $\geq 4$ out of 5 criteria (elevated triglycerides, total and LDL-cholesterol, HbA1c and low HDL-cholesterol), they were classified as MU $(n=52)$. If levels were within reference ranges for $\geq 3$ of the same criteria, subjects were classified as $\mathrm{MH}(n=150)$. Utilizing the entire population, a score ranging from 0 to 5 denoting the number of MU criteria met was computed. Estimated desaturase activities were calculated as productto-precursor ratio of fatty acids in whole blood (SCD16 [16:1n7/16:0], SCD18 [18:1n9/18:0], D5D [18:3n6/18:2n6], D6D $[20: 4 n 6 / 20: 3 n 6])$.
\end{abstract}

Results: Individuals with MH had lower estimated SCD16 and SCD18 activities, whereas estimated D6D activity was higher compared to MU. Similar, SCD16 and SCD18 increased, whereas D6D decreased with increasing criteria of MU. Trends were similar across BMI categories.

Conclusions: This study supports the notion of estimated desaturase activities as possible novel biomarkers of metabolic health irrespectively of BMI.

Keywords: Fatty acids, Desaturases, Obesity, Metabolic disease, Stearoyl CoA desaturase, SCD1, D5D, D6D

\section{Introduction}

Cardiometabolic disorders, including type 2 diabetes (T2D) and cardiovascular disease (CVD) remain the major contributors to the global burden of disease [1]. Obesity is an important risk factor for cardiometabolic disorders, and is thus associated with insulin resistance,

\footnotetext{
* Correspondence: vtelle@oslomet.no

'Svendsen, Karianne and Olsen, Thomas contributed equally and thus share first authorship

${ }^{4}$ Faculty of Health Sciences, Oslo Metropolitan University, P.O. Box 4 St Olavs Plass, 0130 Oslo, Norway

Full list of author information is available at the end of the article
}

glucose intolerance and dyslipidemia [2]. However, not all obese individuals fit into this traditional phenotype, and a subgroup has been described with metabolically healthy $(\mathrm{MH})$ obesity $[3,4]$. Compared with metabolically unhealthy (MU) obesity, the $\mathrm{MH}$ obesity phenotype has a favorable lipid profile and a normal to slightly reduced insulin sensitivity, despite similar body mass index (BMI). Comparable patterns have been observed among individuals with overweight (BMI $25-30 \mathrm{~kg} / \mathrm{m}^{2}$ ). Normal weight individuals (BMI $20-25 \mathrm{~kg} / \mathrm{m}^{2}$ ) are generally considered $\mathrm{MH}$, but $\mathrm{MU}$ normal weight individuals also 
exist $[5,6]$. In an American population, about 2/3 were considered MU despite normal weight [7].

The fatty acid (FA) profile in the circulation is influenced by dietary and lifestyle factors [8, 9], and an unfavorable FA profile has been suggested to predict risk of T2D, CVD and the metabolic syndrome (MetS) [10-14]. Some FAs can be produced endogenously through de novo lipogenesis [15]. Stearoyl-CoA desaturase-1 (SCD1) is a rate-limiting enzyme in catalyzing the desaturation of monounsaturated fatty acids (MUFAs) from saturated fatty acids (SFAs). Delta-5-desaturase (D5D) and delta-6desaturase (D6D) catalyze production of polyunsaturated fatty acids (PUFAs) (Table 1).

An altered FA profile with a high proportion of C16:0 (palmitic acid) increased estimated SCD1 and D6D activities and decreased levels of C18:2n6 FA (linoleic acid) and estimated D5D activity have been associated with insulin resistance, CVD, obesity and development of MetS [16, 17]. Particularly the positive correlation between SCD1 activity, insulin resistance and fat mass has been observed both in animal models and in large human cohorts where SCD1 activity is estimated as product-to-precursor ratios in blood [17-19]. However, it is not known whether alterations in plasma FA profile and desaturase activity is associated with metabolic phenotypes independently of body mass. Hence, the objective of this study was to advance our understanding of the role of FA profile and estimated desaturase activities for the metabolic risk profile ( $\mathrm{MH}$ and $\mathrm{MU}$ ) in otherwise healthy subjects over different BMI categories.

\section{Methods}

\section{Study population}

The population was obtained from the Vascular lifestyleIntervention and Screening in phArmacies (VISA)-study previously described in detail [20]. In short, a subset of 1318 individuals were screened and 582 with moderately elevated CVD risk participated in an 8-week randomized controlled trial (RCT) of lifestyle changes [21]. Of the 582 participants, 321 individuals were followed up one year after the RCT, and were accordingly included in the present cross-sectional study.

Subjects were $\geq 18$ years and had BMI between 18.5 and $44.4 \mathrm{~kg} / \mathrm{m}^{2}$. Importantly, despite moderately elevated risk factors for CVD, all subjects were free of CVD and did not use any medications known to affect serum levels of lipids, glucose, blood pressure or inflammatory markers. Subjects were characterized according to metabolic health phenotypes based mainly on the criteria of Karelis et al. [22]. Subjects were considered MH when fulfilling at least three of the following five criteria: fasting triglycerides $<1.7 \mathrm{mmol} / \mathrm{l}$ or $<2.1 \mathrm{mmol} / \mathrm{l}$ non fasting, total cholesterol $<5.2 \mathrm{mmol} / \mathrm{L}$, low density lipoprotein-cholesterol (LDL-C) $<2.6 \mathrm{mmol} / \mathrm{L}$, high density lipoprotein-cholesterol (HDL-C) $>1.3 \mathrm{mmol} / \mathrm{L}$ and hemoglobin Ab1c (HbA1c) $<5.7 \%$. Subjects were considered MU when fulfilling at least four out of the five following criteria: triglycerides $\geq 1.7 \mathrm{mmol} / \mathrm{L}$ or $\geq 2.1$ $\mathrm{mmol} / \mathrm{L}$ (if not fasting), total cholesterol $\geq 5.2 \mathrm{mmol} / \mathrm{L}$, $\mathrm{LDL}-\mathrm{C} \geq 2.6 \mathrm{mmol} / \mathrm{L}, \mathrm{HDL}-\mathrm{C} \leq 1.3 \mathrm{mmol} / \mathrm{L}$ and $\mathrm{HbA} 1 \mathrm{c}$ $\geq 5.7 \%$. Using measured weight and height, $\mathrm{BMI}$ was calculated as weight $(\mathrm{kg})$ divided by the square of height $\left(\mathrm{m}^{2}\right)$. We defined normal weight, overweight and obese $\left(\mathrm{BMI} \geq 30 \mathrm{~kg} / \mathrm{m}^{2}\right)$, according to the definition by World Health Organization [23]. To make use of data from the entire population, we computed a score based on criteria for MU ranging from 0 to 5 depending on the number of criteria met.

\section{Anthropometric measures}

Data collection was performed by pharmacy staff (pharmacists, technicians or nurses), in accordance with standard operational procedures [20]. Prior to the study, pharmacy staff completed a training program consisting of online e-learning courses and practical training. Subjects were weighed in light clothing without shoes using a digital scale. Height was measured with standing, erect posture and feet against the wall with a wall mounted height board. Blood pressure was measured twice after

Table 1 Stearoyl-CoA desaturase-1 (SCD1), Delta-5-desaturase (D5D) and Delta-6-desaturase (D6D) with purpose, precursor, product and estimated desaturase activity

\begin{tabular}{|c|c|c|c|c|}
\hline $\begin{array}{l}\text { Desaturase enzyme } \\
\text { name }\end{array}$ & Purpose & Precursor & Product & $\begin{array}{l}\text { Estimated desaturase } \\
\text { activity }\end{array}$ \\
\hline SCD1 & $\begin{array}{l}\text { Desaturation of monounsaturated } \\
\text { fatty acids from saturated fatty acids }\end{array}$ & & & \\
\hline SCD16 & & C16:0 (palmitic acid) & $\begin{array}{l}\text { C16:1n7 (palmitoleic } \\
\text { acid) }\end{array}$ & $16: 1 n 7 / 16: 0$ \\
\hline SCD18 & & C18:0 (stearic acid) & C18:1n9 (oleic acid) & $18: 1 n 9 / 18: 0$ \\
\hline D6D & $\begin{array}{l}\text { Desaturation of polyunsaturated fatty } \\
\text { acids (PUFAs) }\end{array}$ & C18:2n6 (linoleic acid) & C18:3n6 ( $y$-linolenic acid) & $18: 3 n 6 / 18: 2 n 6$ \\
\hline D5D & $\begin{array}{l}\text { Desaturation of polyunsaturated fatty } \\
\text { acids (PUFAs) }\end{array}$ & $\begin{array}{l}\text { C20:3n6 (dihomo-y-linolenic } \\
\text { acid) }\end{array}$ & $\begin{array}{l}\text { C20:4n6 (arachidonic } \\
\text { acid) }\end{array}$ & $20: 4 n 6 / 20: 3 n 6$ \\
\hline
\end{tabular}


resting for about five minutes, using A\&D Medical blood pressure Monitor $^{\mathrm{Tw}}$ Model US767Plus30 [21].

\section{Biochemical measures}

HbA1c, total cholesterol, HDL-C and triglycerides were measured by finger-pricks blood samples (Alere Afinio$\left.\mathrm{n}^{\mathrm{m}} \mathrm{AS} 100\right)$. At triglycerides $>7.34 \mathrm{mmol} / \mathrm{L}, \mathrm{HDL}-\mathrm{C}$ could not be measured. LDL-C was calculated using Friedewald's formula. LDL-C was not calculated at triglycerides $>4.52 \mathrm{mmol} / \mathrm{l}$. Non-HDL-C was calculated as total cholesterol - HDL-C [21].

\section{Fatty acid composition in whole blood and desaturase estimation}

FA profile was obtained from whole blood by using dried blood spots (DBS) (VITAS $^{\text {TM }}$ Analytical Services) [24]. DBS is a bio-sampling method where capillary blood, obtained by a finger-prick lancet, are dripped onto spots on filter collection cards (DBS card) [25]. The completed DBS card dried in room temperature for two to four hours before it was stored in an aluminium bag at 1-4 degrees Celsius until shipment to VITAS (Oslo, Norway) for analysis. Fatty acids methyl esters (FAME) were extracted from whole blood (plasma and cells) [26], and were analysed using Gas Chromatography - Flame Ionization Detector (GC-FID) after direct transmethylation. Whole blood FA profile was given in \% FAME. The desaturase activities were estimated as the product-toprecursor ratios of FAs in whole blood according to the following: $\mathrm{SCD} 1=\mathrm{C} 16: 1 \mathrm{n} 7 / \mathrm{C} 16: 0$ and $\mathrm{C} 18: 1 \mathrm{n} 9 / \mathrm{C} 18: 0$, hereafter referred to as SCD16 and SCD18, respectively. $\mathrm{D} 5 \mathrm{D}=\mathrm{C} 18: 3 \mathrm{n} 6 / \mathrm{C} 18: 2 \mathrm{n} 6$ and $\mathrm{D} 6 \mathrm{D}=\mathrm{C} 20: 4 \mathrm{n} 6 / \mathrm{C} 20: 3 \mathrm{n} 6$ (Table 1).

\section{Questionnaires}

From the validated, short food frequency questionnaire (FFQ), VISA-FFQ [27], we obtained data on intake of specific food groups and duration (minutes) and intensity (moderate and vigorous) of physical activity per week. Data on age, sex, smoking habits, marital status, ethnicity, educational level and income had been reported previously ( -52 weeks) through the baseline questionnaire [21].

\section{Statistical analysis}

Baseline characteristics were given as means \pm standard deviations (SD) or median and 25th and 75th percentiles for continuous variables, and counts (\%) for categorical variables. T-tests were used to assess differences in continuous variables, whereas Chi-Square tests were used for categorical variables. Non-normal variables were logtransformed before analysis.

Differences in FA profiles and estimated desaturase activities between the MU and MH groups were assessed using linear regression models adjusted for age and gender. Values are reported as estimated marginal means and corresponding 95\% confidence intervals (CI). Furthermore, the association of FA profiles and estimated desaturase activities with increasing numbers of criteria for MU were investigated using linear regression adjusted for age and gender. We further explored whether trends were similar in BMI categories by adding an interaction term to the models (BMI $\times$ metabolic status) to assess whether the association of metabolic status with FA profiles depended on BMI. Finally, we used principal component analysis (PCA) to explore whether clusters of whole blood FAs were associated with $\mathrm{MH}$ and MU. All individual FAs were selected and normalized (scaled and centred) prior to PCA. Scree plots were examined and revealed three components explaining $52 \%$ of the total variance in the dataset after application of varimax rotation. We proceeded with principal component (PC) 1 and $\mathrm{PC} 2$ and the computed PC scores were scaled and centred and given as z-scores. The PCA procedure was performed using the psych package for $\mathrm{R}$. Linear regression was used to assess differences in PC1 and PC2 between MU and MH. A $p$-value of $<0.05$ was considered statistically significant. We opted to not adjust for multiplicity due to the exploratory nature of the study. Statistical analyses were carried out using IBM SPSS version 23 and $R$ version 3.6.3, using packages included in the "tidyverse" package selection, "psych" and "princomp". Plots were made with the ggplot2 package for R.

\section{Results}

Metabolic health, fatty acid profile and estimated desaturase activities

Characterization of the participants is presented in Table 2. Of 321 subjects, $37.1 \%(n=119)$ were categorized as normal weight, $40.2 \%(n=129)$ were overweight and $22.7 \%(n=73)$ were categorized as obese. Of these, 202 subjects met the criteria for $\mathrm{MH}$ and $\mathrm{MU}$, and were subsequently categorized as $\mathrm{MH}(n=150)$ or $\mathrm{MU}(n=$ 52). The individuals with $\mathrm{MH}$ phenotype were slightly younger ( $55.5 \pm 14.8$ years vs. $60.5 \pm 12.1$ years) and reported higher physical activity (median $[25,75$ th percentile]: $231 \quad(79.3,469)$ minutes vs. $114(0,247)$ minutes) than individuals with MU phenotype. There were no differences in blood pressure, gender, years of education, or prevalence of smokers between $\mathrm{MH}$ and MU subjects (Table 2).

Of the FAs measured, C18:0 was higher, whereas C14: 0 and $\mathrm{C} 16: 0$ were lower in the $\mathrm{MH}$ group compared with MU group. Furthermore, the $\mathrm{MH}$ group had lower levels of total MUFAs, C16:1n7, C18:1n9 and estimated SCD16 and SCD18 activity compared to the MU group $(P<0.001$ for all). Except for C18:3n3, total n6 FA, n3 
Table 2 Characteristics of metabolically healthy and unhealthy subjects

\begin{tabular}{|c|c|c|c|}
\hline & Metabolically healthy $(n=150)$ & Metabolically unhealthy $(n=52)$ & $P$ \\
\hline \multicolumn{4}{|l|}{ Demographics } \\
\hline Age, years (mean $\pm(S D)$ ) & $55.5 \pm 14.8$ & $60.5 \pm 12.1$ & 0.017 \\
\hline Men, n (\%) & $34(22.7)$ & 19 (36.5) & $0.132^{1}$ \\
\hline$\leq 13$ years of schooling, $\mathrm{n}(\%)$ & $77(54.6)$ & $26(51.0)$ & $0.854^{1}$ \\
\hline Smokers (daily or occasional), n (\%) & $18(12.3)$ & $5(9.6)$ & $0.607^{1}$ \\
\hline \multicolumn{4}{|l|}{ Risk factors (mean $\pm(S D)$ ) } \\
\hline $\mathrm{BMl}, \mathrm{kg} / \mathrm{m}^{2}$ & $26.6 \pm 4.6$ & $28.8 \pm 4.5$ & 0.003 \\
\hline Total cholesterol, mmol/l & $6.2 \pm 1.1$ & $6.7 \pm 1.0$ & 0.012 \\
\hline LDL-cholesterol, mmol// & $3.8 \pm 1.0$ & $4.0 \pm 0.9$ & 0.073 \\
\hline HDL-cholesterol, mmol// & $1.9 \pm 0.5$ & $1.4 \pm 0.4$ & $<0.001$ \\
\hline $\mathrm{NonHDL}, \mathrm{mmol} / \mathrm{l}$ & $4.3 \pm 0.9$ & $5.2 \pm 0.9$ & $<0.001$ \\
\hline Triglycerides, mmol/l & $1.3 \pm 0.5$ & $2.9 \pm 1.1$ & $<0.001$ \\
\hline $\mathrm{HbA} 1 \mathrm{c}, \%$ & $5.4 \pm 0.2$ & $5.8 \pm 0.4$ & $<0.001$ \\
\hline Systolic BP, mmHg & $126.2 \pm 16.1$ & $126.6 \pm 16.8$ & 0.857 \\
\hline Diastolic BP, mmHg & $79.5 \pm 9.9$ & $80.4 \pm 10.1$ & 0.567 \\
\hline Physical activity, min/week ${ }^{2,3}$ & $231(79.3,469)$ & $114(0,247)$ & 0.002 \\
\hline
\end{tabular}

Demographics as frequency [n (\%)] and age were obtained 52 weeks prior to the present data collection. Risk factors are expressed as mean \pm SD. $P$ values from Student's t-test. $P$ is significant at 0.05 level. ${ }^{1} P$ value from Chi-square test. BMI, body mass index; BP, blood pressure; HbA1c, glycated hemoglobin A1c. ${ }^{2}$ Given as median (25th - 75th percentile). ${ }^{3}$ Mann-Whitney $\mathrm{U}$ test

FA and D6D, but not D5D, were significantly higher in the $\mathrm{MH}$ group (Table 3). We observed that estimated SCD16 and SCD18 activities ( $P$ for both $<0.001)$ increased with increasing number of criteria for MU (ranging 1-5). A clear decrease was observed for estimated D6D activity $(P<0.001)$ and a slight decrease was observed for estimated D5D activity with increasing number of criteria for MU $(P=0.03)$ (Fig. 1).

\section{Principal component analyses}

In order to explore whether clusters of FAs were different between the $\mathrm{MH}$ and MU groups, we performed PCA. All individual FAs were selected for analysis. PC1 and PC2 accounted for $\sim 36 \%$ of the total variance in the dataset. Briefly, the FAs most positively correlated with $\mathrm{PC} 1$ were $\mathrm{C} 20: 5 \mathrm{n} 3, \mathrm{C} 22: 5 \mathrm{n} 3$ and $\mathrm{C} 22: 6 \mathrm{n} 3$ whereas $\mathrm{C} 18$ : 1n9 were strongly and inversely correlated with PC1. For PC2, the strongest positive correlations were observed for $\mathrm{C} 16: 0$ and $\mathrm{C} 16: 1 \mathrm{n} 7$ and inverse correlations for $\mathrm{C} 18$ : 2n6 and C18:0. Compared with $\mathrm{MH}$, individuals with MU had a lower mean z-score for PC1 and a higher mean z-score for PC2 as illustrated in Fig. 2.

\section{Association of metabolic health phenotypes and estimated desaturases by BMI category}

We further investigated the MU phenotypes according to BMI categories. Characteristics of $\mathrm{MH}$ and $\mathrm{MU}$ individuals by BMI group are presented in Additional file 1 .

Of the overall population of individuals with obesity (including those between MU and MH), 46.6\% ( $n=34)$ were categorized as $\mathrm{MH}$ (corresponding to $10.4 \%$ of the total population) and $23.3 \%(n=17)$ as MU. The corresponding prevalence of $\mathrm{MH}$ among the individuals with overweight were $40.3 \%(n=52)$ and $17.8 \% \mathrm{MU}(n=23)$. Among those with normal weight, $53.8 \%(n=64)$ were MH and 9.2\% $(n=11)$ were MU. Parameters were generally similar except for the metabolic status classification criteria. Individuals with $\mathrm{MH}$ and obesity reported higher physical activity with median (25th-75th percentile) $150 \mathrm{~min}(0,362)$ per week compared with $38 \mathrm{~min}(0$, 126) for MU with obesity $(P=0.031)$. The FA profile and estimated desaturase activities were similar between MU and $\mathrm{MH}$ subjects in each BMI category and no interactions were observed in the regression models (Additional file 2). The association between numbers of criteria met for MU overall showed the same patterns in each BMI category as for the total population (Additional file 3).

\section{Discussion}

In a group of Norwegians with moderately elevated risk of CVD but non-medically treated, we found that estimated SCD16 and SCD18 activities increased with increasing number of criteria for $\mathrm{MU}$, whereas estimated D5D and D6D activities appeared to decrease. Similar, some SFAs, total MUFA, estimated SCD16 and SCD18 were lower, whereas most PUFAs (both $n 3$ and n6) and D6D were higher in $\mathrm{MH}$ than $\mathrm{MU}$. These findings were also supported by results from PCA. Related findings were observed in subgroups according to BMI. 
Table 3 Fatty acid profile and estimated desaturase activity in metabolically healthy and unhealthy subjects

\begin{tabular}{|c|c|c|c|}
\hline Fatty acids, \%FAME & Metabolically healthy $(n=150)$ & Metabolically unhealthy $(n=52)$ & $P$ \\
\hline C12:0 & $0.12(0.1,0.14)$ & $0.14(0.12,0.17)$ & 0.169 \\
\hline C14:0 & $0.91(0.85,0.98)$ & $1.16(1.06,1.25)$ & $<0.001$ \\
\hline C15:0 & $0.25(0.24,0.26)$ & $0.25(0.23,0.26)$ & 0.658 \\
\hline C16:0 & $21.51(21.25,21.78)$ & $22.35(21.95,22.76)$ & 0.001 \\
\hline C17:0 & $0.35(0.34,0.36)$ & $0.33(0.32,0.35)$ & 0.049 \\
\hline C18:0 & $11.79(11.62,11.96)$ & $10.98(10.72,11.24)$ & $<0.001$ \\
\hline C20:0 & $0.09(0.08,0.09)$ & $0.09(0.09,0.1)$ & 0.167 \\
\hline SFA total & $35.03(34.72,35.34)$ & $35.3(34.84,35.77)$ & 0.323 \\
\hline C16:1 n7 & $1.36(1.26,1.46)$ & $1.72(1.57,1.86)$ & $<0.001$ \\
\hline C18:1 n9 & $19.85(19.45,20.24)$ & $22.51(21.9,23.11)$ & $<0.001$ \\
\hline $\mathrm{C} 18: 1 \mathrm{c} 11$ & $1.54(1.49,1.59)$ & $1.51(1.44,1.59)$ & 0.512 \\
\hline C20:1 n9 & $0.26(0.24,0.27)$ & $0.24(0.22,0.26)$ & 0.221 \\
\hline MUFA total & $23(22.55,23.45)$ & $25.98(25.3,26.66)$ & $<0.001$ \\
\hline SCD16 & $0.06(0.06,0.07)$ & $0.08(0.07,0.08)$ & $<0.001$ \\
\hline SCD18 & $1.7(1.64,1.75)$ & $2.08(2,2.16)$ & $<0.001$ \\
\hline D5D & $0.01(0.01,0.01)$ & $0.01(0.01,0.01)$ & 0.081 \\
\hline D6D & $5.87(5.65,6.09)$ & $5.14(4.8,5.48)$ & $<0.001$ \\
\hline C18:2 n6 & $19.81(19.34,20.29)$ & $18.94(18.22,19.66)$ & 0.042 \\
\hline C18:3 n6 & $0.2(0.18,0.22)$ & $0.22(0.19,0.24)$ & 0.257 \\
\hline C18:3 n3 & $0.49(0.46,0.52)$ & $0.61(0.56,0.66)$ & $<0.001$ \\
\hline C20:3 n6 & $1.4(1.35,1.45)$ & $1.38(1.31,1.46)$ & 0.694 \\
\hline C20:4 n6 & $8.03(7.77,8.29)$ & $7.01(6.61,7.4)$ & $<0.001$ \\
\hline$C 20: 5$ n3 & $1.48(1.33,1.63)$ & $1.06(0.83,1.29)$ & 0.002 \\
\hline C22:5 n3 & $1.39(1.34,1.44)$ & $1.21(1.13,1.28)$ & $<0.001$ \\
\hline $\mathrm{C} 22: 6 \mathrm{n} 3$ & $3.4(3.25,3.56)$ & $2.98(2.74,3.21)$ & 0.003 \\
\hline PUFA total & $36.21(35.63,36.79)$ & $33.4(32.52,34.28)$ & $<0.001$ \\
\hline$\omega-3$ total & $6.77(6.46,7.08)$ & $5.85(5.37,6.33)$ & 0.001 \\
\hline$\omega-6$ total & $29.44(28.91,29.98)$ & $27.55(26.74,28.35)$ & $<0.001$ \\
\hline
\end{tabular}

$P$ values from linear regression models adjusted for age and sex. Values are estimated marginal means $95 \%$ confidence interval). $P$ is significant at 0.05 level. SCD1, Stearoyl-CoA desaturase-1; D5D, delta-5-desaturase; D6D, delta-6-desaturase; SFA, saturated fatty acids; MUFA, monounsaturated fatty acids; PUFA, polyunsaturated fatty acids

Findings from this study suggest that all of the investigated desaturases were related to metabolic health. The association between criteria for MU and desaturases was strongest for estimated SCD16 and SCD18 activities. In a subsample in the PREDIMED trial, estimated SCD16 but not SCD18 activity was increased in individuals with MetS [28]. Such an association may primarily be explained by obesity according to Warensjo et al. [12]. Additionally, findings from one large cohort study suggested that both SCD16 and SCD18 activities were associated with fat mass [18], further supporting that obesity can be a main driver of these associations. However, we observed associations between SCD activities and MU irrespectively of BMI subgroups. In line with our results, Lassale et al. found that individuals with a MU phenotype had higher risk of CVD than $\mathrm{MH}$, irrespective of BMI [29]. One possible explanation for our findings could be different dietary intakes of SFAs, as estimated SCD16 activity has been shown to be higher after intervention with a diet high in SFA compared to a diet high in MUFA and PUFA $[30,31]$. However, in the present study, we observed that intake of foods high in SFA did not differ between $\mathrm{MH}$ and MU (Additional file 4).

In addition to lower plasma levels of the SFAs C14:0 and $\mathrm{C} 16: 0$, we also found lower levels of $\mathrm{C} 16: 1 \mathrm{n} 7$ and $\mathrm{C} 18: 1 \mathrm{n} 9$ in $\mathrm{MH}$ subjects, including $\mathrm{MH}$ obese compared with their MU counterparts. This finding corresponds to the lower levels of estimated SCD16 and SCD18 activities in $\mathrm{MH}$, as endogen production of MUFAs is regulated by these desaturases [15].

MUFAs (C16:1 and 18:1) are major components of triglycerides, cholesterol esters and phospholipids, 


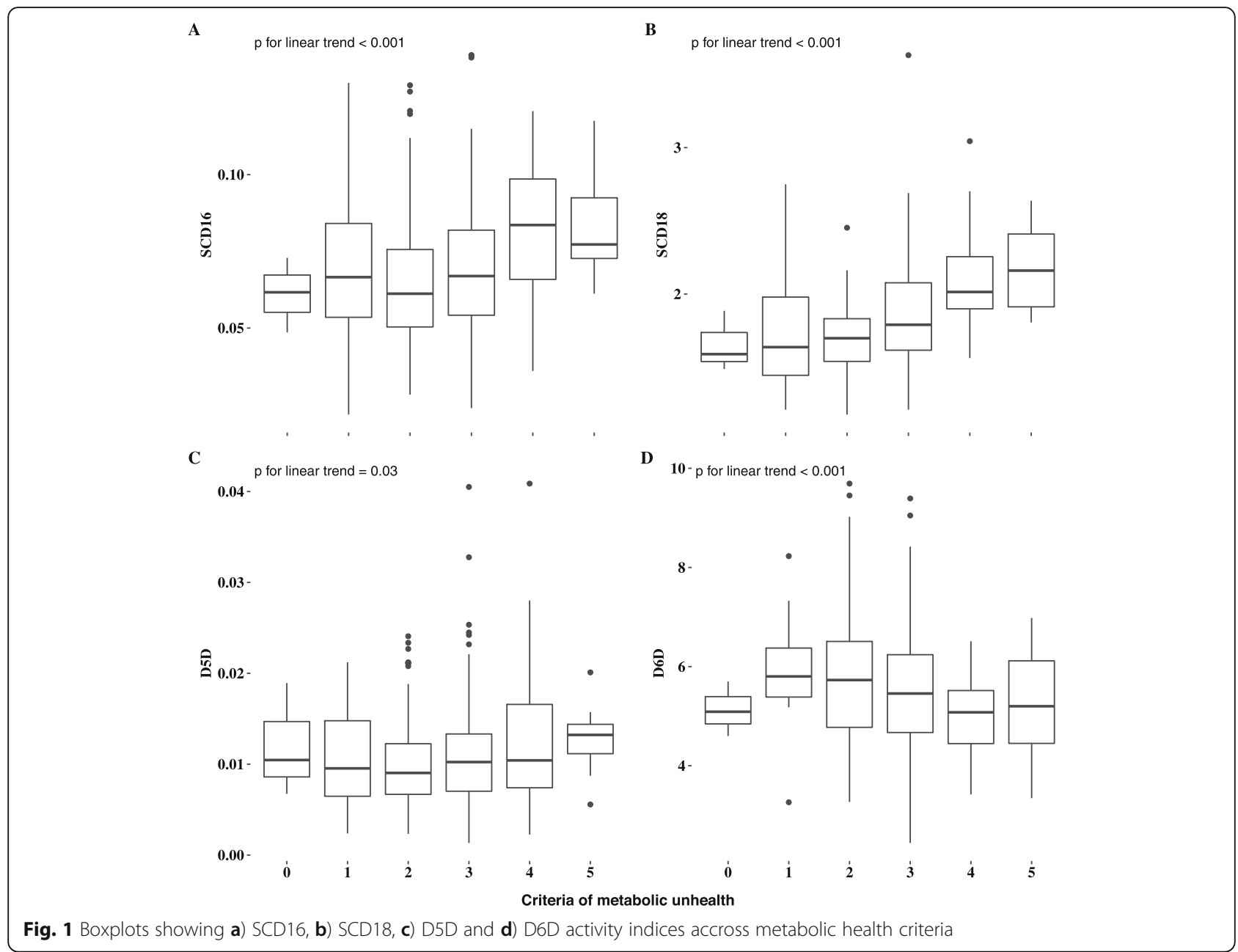

produced by de novo lipogenesis and carried in VLDL in the circulation [15]. This incorporation of MUFAs in VLDL, could partly explain the observed association between high levels of MUFAs and the MU phenotype, characterized by high levels of triglycerides, total cholesterol and LDL-C. Our findings are also supported by results from a NHANES survey where higher plasma concentration of SFAs and MUFAs were associated with increased levels of fasting plasma glucose and HbA1c [32]. The same pattern was found in the FINRISK study. Here, higher plasma levels of MUFAs in healthy, overweight subjects were associated with increased CVD risk, whereas higher levels of n6 FAs and docosahexaenoic acid (DHA) levels were associated with lower risk [33], in line with our study.

We found that the estimated activity of enzymes responsible for the desaturation of PUFAs, D6D and to some extent D5D activities, decreased with increasing numbers of criteria for MU. Both Zhao et al. and Mayneris-Perxachs et al. found that estimated D5D activity was inversely associated with metabolic dysregulation [28, 34], also in $\mathrm{MH}$ obese subjects [34]. The observed higher levels of estimated D6D in MH subjects in our study was therefore in contrast to other findings where D6D has been associated with increased risk of MetS and insulin resistance in individuals with overweight and obesity [28, 35]. The association has been suggested to be due to PUFA imbalance such as reduced n3/n6 FAs ratio [35]. However, the ratio in blood between $n 3$ and $n 6$ in the present study was not excessively high [35]. Neither where there any differences in dietary intake of fatty fish (high in n3 FA) between the $\mathrm{MH}$ and MU group.

Our findings suggests that SCD may influence the development of MU phenotype independently of fat mass and dietary intake. Consequently, other unmeasured metabolic markers could affect the association between SCD and MU phenotype. Genetics may play an important role, as several studies in rodents have shown that SCD16 deficient mice do not become overweight/obese or have metabolic disruptions even when fed a high fat diet [36]. In this regard, body fat distribution among subjects and across genders is another unmeasured factor that could have helped elucidated this results [37]. 

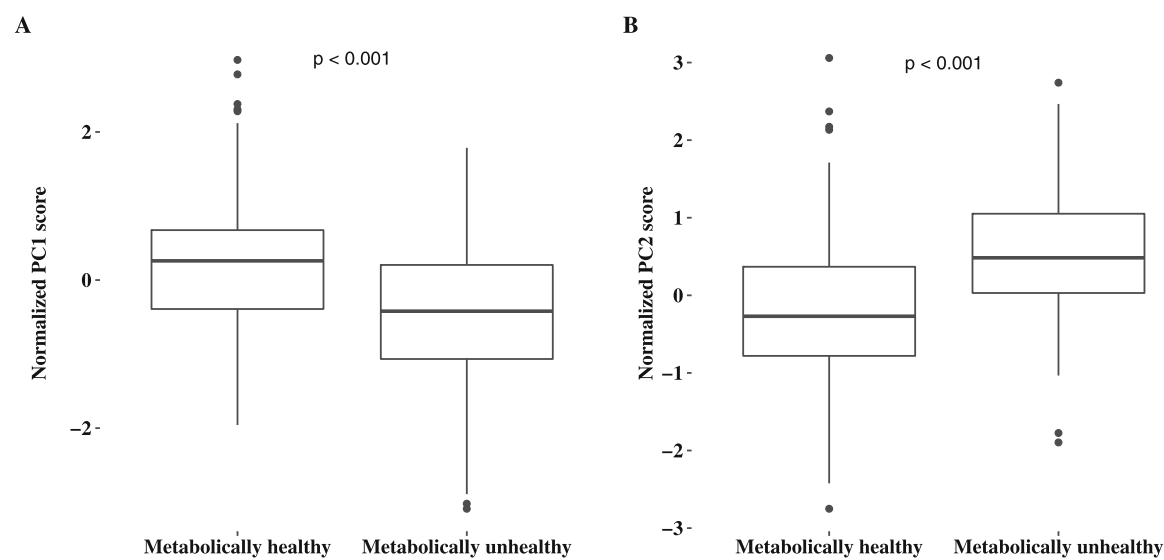

C

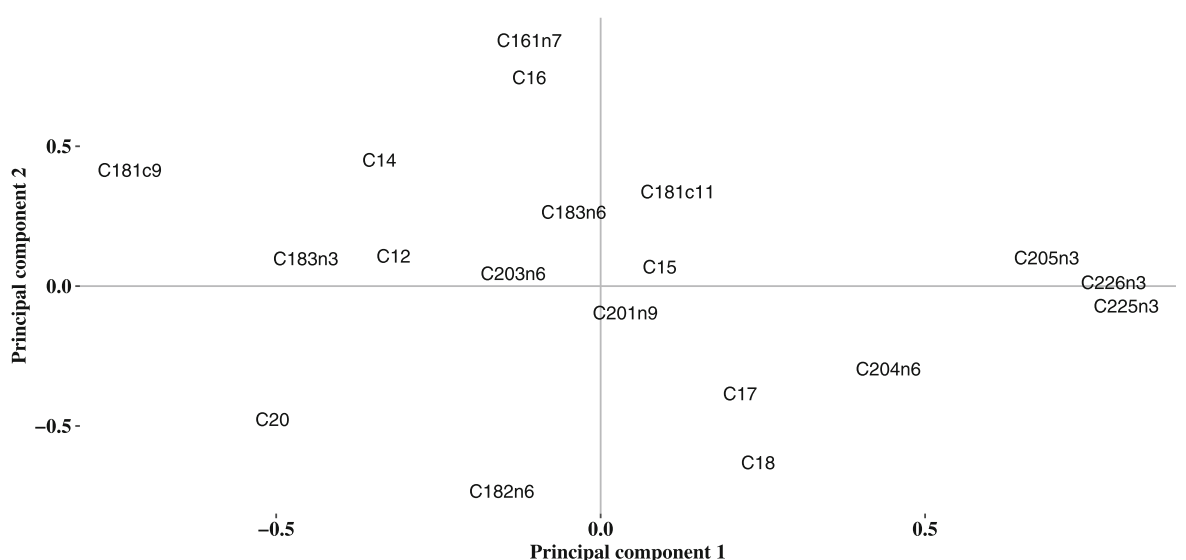

Fig. 2 a) Boxplot for principal component 1 score, b) boxplot for principal component 2 score aand c) correlation between the individual fatty acids and prinicpal component 1 and 2

For instance, $\mathrm{MH}$ overweight and obese could have lower levels of visceral and ectopic fat [38, 39]. Furthermore, we observed $\mathrm{MH}$ to be more physically active than MU inn all BMI groups, indicating that lifestyle is likely to be important for metabolic health.

It is however possible that our study population is healthier than other populations. In total $10 \%$ defined as $\mathrm{MH}$ obese is among the highest prevalence independent of criteria used for metabolic health summarized by Liu et al. [40] and Wildman et al. [41]. Using BMI rather than waist circumference to define obesity often yields higher prevalence of $\mathrm{MH}$ obese [40]. Yet, other studies have found comparable prevalence of $\mathrm{MH}$ obese as the present study using $\geq 2$ criteria for metabolic health [42]. Furthermore, subjects were enrolled in the present study based on background CVD risk and had previously attended an intervention study aiming at changing lifestyle behavior [21]. This might have influenced the study population and limiting the generalizability of results.

In line with this, we observed that in the present study, $9 \%(n=13)$ of the MH were previously (1 year before) categorized as MU. This transition from MU to $\mathrm{MH}$ was due to favorable changes in diet, lifestyle and CVD risk factors previously described as long-term effects of participating in the VISA-study [21]. On another note, it has been suggested that $\mathrm{MH}$ overweight and obese phenotypes may be transient states, as individuals with $\mathrm{MH}$ overweight and obesity are still at increased risk of developing T2D and CVD compared to normal weight individuals [43]. FAs and desaturase activity indices could therefore be predictors of long-term development of metabolic disorders [12], such as a 10-year transition from $\mathrm{MH}$ to $\mathrm{MU}$ [34]. Hence, our results support that desaturases could be biomarkers of (future) metabolic health independent of BMI, although direction of association remains to be elucidated.

\section{Strengths and limitations}

The main strength of the study is the well-characterized sample of 321 individuals free of disease but at moderately increased risk of CVD who were originally recruited from the general population [20, 21]. Limitations 
includes lack of generalizability, as the study population had been previously included in an intervention study aiming at improving health. Furthermore, we note that due to the cross-sectional nature of the study, the direction of the associations reported is impossible to infer and no information on causality can be obtained.

\section{Conclusion}

We found that estimated SCD16 and SCD18 activities increased with increasing number of criteria for $\mathrm{MU}$, and that estimated D6D activity and to some extent also D5D activity, decreased. The corresponding SFAs and PUFAs followed the patterns of the estimates activities of SCD16/SCD18 and D5D/D6D respectively. The findings persisted irrespectively of BMI. Hence, the study supports that these desaturases can be important novel biomarkers of metabolic health, although these analyses cannot answer whether the dysregulation of these desaturases are a cause or a consequence of lifestyle factors or genetics. This warrants further studies.

\section{Supplementary information}

Supplementary information accompanies this paper at https://doi.org/10. 1186/s12944-020-01282-y.

Additional file 1. Characteristics of metabolically healthy and unhealthy normal weight, overweight and obese subjects.

Additional file 2. Fatty acid profile and desaturase ratios in metabolically healthy and unhealthy normal weight, overweight and obese subjects.

Additional file 3. Association of criteria for metabolic health and estimated desaturase activities in BMI categories.

Additional file 4. Diet and lifestyle for metabolically healthy $(\mathrm{MH})$ and unhealthy (MU) participants stratified by body mass index (BMI).

\section{Abbreviations \\ CVD: Cardiovascular diseases; FA: Fatty acid; HDL: High density lipoprotein; HbA1c: Hemoglobin A1c; LDL: Low density lipoprotein; MetS: Metabolic syndrome; $\mathrm{MH}$ : Metabolic healthy; MU: Metabolic unhealthy; MUFA: Monounsaturated fatty acids; PUFA: Polyunsaturated fatty acids; SCD1: Stearoyl CoA-desaturase 1; SFA: Saturated fatty acids; T2D: Type 2 diabetes}

\section{Acknowledgements \\ We are grateful for all participants in the present study and the VISA-study. Our sincerely thanks to employees in Boots Norge AS for collecting both biochemical measurements and dried blood spot tests. Thanks to Kjersti W. Garstad and Lisa T. Mørch-Reiersen in Boots Norge AS and David R. Jacobs Jr. from University of Minnesota for significant contributions to study administration and to the design of the VISA-study. Thanks to Hege Berg Henriksen for assisting in development and processing of the questionnaire used to report physical activ- ity level.}

\section{Authors' contributions}

KS, KR, VTH were responsible for study administration. KS, TO, TCNR, SMU, $\mathrm{KBH}, \mathrm{KR}$ and $\mathrm{VTH}$ contributed to the conceptualization of the study. KS, TO, TCNR, KR and VHTH and were responsible for data curation, and TO and TCNR for the main data analysis. KS, TO and VHTH were responsible for writing the main manuscript text. All authors reviewed and approved the final manuscript.

\section{Funding}

We received funding from Wendel Jarlsbergs (distributed by the non-profit organization UNIFOR) for analysis of fatty acids and development of the VISA-FFQ questionnaire used to report diet and physical activity. We also previously received funding from Vita hjertego' (Mills DA) for work with the VISA-FFQ.

Availability of data and materials

The datasets used and/or analysed during the current study are available from the corresponding author on reasonable request.

Ethics approval and consent to participate

The study was approved by the Regional Committee of Medical Ethics (ref. 2013/1660). Written informed consent for participation was obtained, and the study complied with the Declaration of Helsinki.

Consent for publication

Not applicable.

\section{Competing interests}

TO and TCNR declare that they have no competing interests. KS reports grants from Mills DA (Vita hjertego') relevant for the present paper, and grants from a pharmacy interest organization (Stiftelsen til fremme av. norsk apotekfarmasi), personal fees from MedXplore outside the submitted work. SMU has received research grants from Tine DA, Mills DA, Olympic Seafood, none of which are related to the content of this manuscript. $\mathrm{KBH}$ reports grants from Tine SA, Mills DA, Olympic Seafood, Amgen, Sanofi, Kaneka and personal fees from Amgen, Sanofi, Pronova, outside the submitted work. KR reports personal fees from MedXplore, Amgen, Mills DA, The Norwegian Medical Association, The Norwegian Directorate of Health, Sanofi, Takeda, Chiesi, Bayer and MSD, outside the submitted work. VHTH is a former employee at Mills AS and reports grants from Mills AS. She does not owns any stocks in the company, and the work performed in this paper was done after she left the company.

\section{Author details}

${ }^{1}$ Department of Nutrition, Institute of Basic Medical Sciences, Faculty of Medicine, University of Oslo, Oslo, Norway. ${ }^{2}$ The Lipid Clinic, Department of Endocrinology, Morbid Obesity and Preventive Medicine, Oslo University Hospital, Oslo, Norway. ${ }^{3}$ National Advisory Unit on Familial Hypercholesterolemia, Department of Endocrinology, Morbid Obesity and Preventive Medicine, Oslo University Hospital, Oslo, Norway. ${ }^{4}$ Faculty of Health Sciences, Oslo Metropolitan University, P.O. Box 4 St Olavs Plass, 0130 Oslo, Norway.

Received: 12 November 2019 Accepted: 8 May 2020

Published online: 21 May 2020

References

1. Global Burden of Disease Mortality Causes of Death Collaborators. Global, regional, and national life expectancy, all-cause mortality, and cause-specific mortality for 249 causes of death, 1980-2015: a systematic analysis for the Global Burden of Disease Study 2015. Lancet. 2016;388(10053):1459-544

2. Ipsen DH, Tveden-Nyborg P, Lykkesfeldt J. Dyslipidemia: obese or not obese-that is not the question. Curr Obes Rep. 2016;5(4):405-12.

3. Telle-Hansen VH, Halvorsen B, Dalen KT, Narverud I, Wesseltoft-Rao N, Granlund $L$, et al. Altered expression of genes involved in lipid metabolism in obese subjects with unfavourable phenotype. Genes Nutr. 2013;8(4):425-34.

4. Munoz-Garach A, Cornejo-Pareja I, Tinahones FJ. Does Metabolically Healthy Obesity Exist? Nutrients. 2016;8(6):1-10.

5. Caleyachetty R, Thomas GN, Toulis KA, Mohammed N, Gokhale KM, Balachandran $\mathrm{K}$, et al. Metabolically healthy obese and incident cardiovascular disease events among 3.5 million men and women. J Am Coll Cardiol. 2017;70(12):1429-37.

6. Aung K, Lorenzo C, Hinojosa MA, Haffner SM. Risk of developing diabetes and cardiovascular disease in metabolically unhealthy normal-weight and metabolically healthy obese individuals. J Clin Endocrinol Metab. 2014;99(2): 462-8.

7. Araujo J, Cai J, Stevens J. Prevalence of optimal metabolic health in American adults: National Health and nutrition examination survey 20092016. Metab Syndr Relat Disord. 2019;17(1):46-52. 
8. Bjermo H, Riserus U. Role of hepatic desaturases in obesity-related metabolic disorders. Curr Opin Clin Nutr Metab Care. 2010;13(6):703-8.

9. Vessby B, Gustafsson IB, Tengblad S, Boberg M, Andersson A. Desaturation and elongation of fatty acids and insulin action. Ann N Y Acad Sci. 2002; 967:183-95.

10. Lankinen MA, Stancakova A, Uusitupa M, Agren J, Pihlajamaki J, Kuusisto J, et al. Plasma fatty acids as predictors of glycaemia and type 2 diabetes. Diabetologia. 2015;58(11):2533-44.

11. Kim OY, Lim HH, Lee MJ, Kim JY, Lee JH. Association of fatty acid composition in serum phospholipids with metabolic syndrome and arterial stiffness. Nutr Metab Cardiovasc Dis. 2013;23(4):366-74

12. Warensjo E, Riserus $U$, Vessby B. Fatty acid composition of serum lipids predicts the development of the metabolic syndrome in men. Diabetologia. 2005:48(10):1999-2005

13. Tremblay AJ, Despres JP, Piche ME, Nadeau A, Bergeron J, Almeras N, et al. Associations between the fatty acid content of triglyceride, visceral adipose tissue accumulation, and components of the insulin resistance syndrome. Metab Clin Exp. 2004;53(3):310-7.

14. Wang L, Folsom AR, Zheng ZJ, Pankow JS, Eckfeldt JH, Investigators AS. Plasma fatty acid composition and incidence of diabetes in middle-aged adults: the atherosclerosis risk in communities (ARIC) study. Am J Clin Nutr. 2003;78(1):91-8

15. Ferrier DR. In: Harvey RA, editor. Biochemistry. Sixth edition; 2014.

16. Riserus U, Willett WC, Hu FB. Dietary fats and prevention of type 2 diabetes. Prog Lipid Res. 2009:48(1):44-51.

17. Warensjo E, Ohrvall M, Vessby B. Fatty acid composition and estimated desaturase activities are associated with obesity and lifestyle variables in men and women. Nutr mMetab Cardiovasc Dis. 2006;16(2):128-36.

18. Vinknes KJ, Elshorbagy AK, Nurk E, Drevon CA, Gjesdal CG, Tell GS, et al. Plasma stearoyl-CoA desaturase indices: association with lifestyle, diet, and body composition. Obesity (Silver Spring, Md). 2013;21(3):E294-302.

19. ALJohani AM, Syed DN, Ntambi JM. Insights into Stearoyl-CoA Desaturase-1 regulation of systemic metabolism. Trends Endocrinol Metab. 2017;28(12): $831-42$

20. Svendsen K, Jacobs DR Jr, Royseth IT, Garstad KW, Byfuglien MG, Granlund L, et al. Community pharmacies offer a potential high-yield and convenient arena for total cholesterol and CVD risk screening. Eur J Public Health. 2018; 29(1):17-23.

21. Svendsen K, Telle-Hansen VH, Morch-Reiersen LT, Garstad KW, Thyholt K, Granlund $\mathrm{L}$, et al. A randomized controlled trial in Norwegian pharmacies on effects of risk alert and advice in people with elevated cardiovascular risk. Prev Med Rep. 2018;12:79-86.

22. Karelis AD, Brochu M, Rabasa-Lhoret R. Can we identify metabolically healthy but obese individuals (MHO)? Diabetes Metab. 2004;30(6):569-72.

23. World Health Organization. Obesity and overweight 2018 [cited 2019 21.02]. Available from: https://www.who.int/news-room/fact-sheets/detail/obesityand-overweight

24. Sakhi AK, Bastani NE, Ellingjord-Dale M, Gundersen TE, Blomhoff R, Ursin G. Feasibility of self-sampled dried blood spot and saliva samples sent by mail in a population-based study. BMC Cancer. 2015;15(1):265.

25. Edelbroek PM, van der Heijden J, Stolk LM. Dried blood spot methods in therapeutic drug monitoring: methods, assays, and pitfalls. Ther Drug Monit. 2009;31(3):327-36

26. Rise P, Eligini S, Ghezzi S, Colli S, Galli C. Fatty acid composition of plasma, blood cells and whole blood: relevance for the assessment of the fatty acid status in humans. Prostaglandins Leukot Essent Fat Acids. 2007;76(6):363-9.

27. Svendsen $\mathrm{K}$, Henriksen HB, Ostengen $\mathrm{B}$, Jacobs DR Jr, Telle-Hansen VH, Carlsen $\mathrm{MH}$, et al. Evaluation of a short food frequency questionnaire to assess cardiovascular disease-related diet and lifestyle factors. Food Nutr Res. 2018;62.

28. Mayneris-Perxachs J, Guerendiain M, Castellote Al, Estruch R, Covas Ml, Fito $M$, et al. Plasma fatty acid composition, estimated desaturase activities, and their relation with the metabolic syndrome in a population at high risk of cardiovascular disease. Clin Nutr (Edinburgh, Scotland). 2014;33(1):90-7.

29. Lassale C, Tzoulaki I, Moons KGM, Sweeting M, Boer J, Johnson L, et al. Separate and combined associations of obesity and metabolic health with coronary heart disease: a pan-European case-cohort analysis. Eur Heart J. 2018;39(5):397-406.

30. Warensjo E, Riserus U, Gustafsson IB, Mohsen R, Cederholm T, Vessby B. Effects of saturated and unsaturated fatty acids on estimated desaturase activities during a controlled dietary intervention. Nutr Metab Cardiovasc Dis. 2008;18(10):683-90.

31. Telle-Hansen VH, Larsen LN, Hostmark AT, Molin M, Dahl L, Almendingen K, et al. Daily intake of cod or salmon for 2 weeks decreases the 18:1n-9/18:0 ratio and serum triacylglycerols in healthy subjects. Lipids. 2012;47(2):151-60.

32. Yu EA, Hu PJ, Mehta S. Plasma fatty acids in de novo lipogenesis pathway are associated with diabetogenic indicators among adults: NHANES 20032004. Am J Clin Nutr. 2018;108(3):622-32.

33. Wurtz P, Havulinna AS, Soininen P, Tynkkynen T, Prieto-Merino D, Tillin T, et al. Metabolite profiling and cardiovascular event risk: a prospective study of 3 population-based cohorts. Circulation. 2015;131(9):774-85.

34. Zhao L, Ni Y, Ma X, Zhao A, Bao Y, Liu J, et al. A panel of free fatty acid ratios to predict the development of metabolic abnormalities in healthy obese individuals. Sci Rep. 2016;6:28418.

35. Arshad Z, Rezapour-Firouzi S, Ebrahimifar M, Mosavi Jarrahi A, Mohammadian M. Association of Delta-6-Desaturase expression with aggressiveness of Cancer, diabetes mellitus, and multiple sclerosis: a narrative review. Asian Pac J Cancer Prev. 2019;20(4):1005-18.

36. Bai Y, McCoy JG, Levin EJ, Sobrado P, Rajashankar KR, Fox BG, et al. X-ray structure of a mammalian stearoyl-CoA desaturase. Nature. 2015;524(7564): 252-6.

37. Frank AP, de Souza SR, Palmer BF, Clegg DJ. Determinants of body fat distribution in humans may provide insight about obesity-related health risks. J Lipid Res. 2019:60(10):1710-9.

38. Karelis AD, St-Pierre DH, Conus F, Rabasa-Lhoret R, Poehlman ET. Metabolic and body composition factors in subgroups of obesity: what do we know? J Clin Endocrinol Metab. 2004;89(6):2569-75.

39. Primeau V, Coderre L, Karelis AD, Brochu M, Lavoie ME, Messier V, et al. Characterizing the profile of obese patients who are metabolically healthy. Int J Obes. 2011;35(7):971-81.

40. Liu C, Wang C, Guan S, Liu H, Wu X, Zhang Z, et al. The prevalence of metabolically healthy and unhealthy obesity according to different criteria. Obesity facts. 2019:12(1):78-90.

41. Wildman RP, Muntner P, Reynolds K, McGinn AP, Rajpathak S, Wylie-Rosett J, et al. The obese without cardiometabolic risk factor clustering and the normal weight with cardiometabolic risk factor clustering: prevalence and correlates of 2 phenotypes among the US population (NHANES 1999-2004). Arch Intern Med. 2008;168(15):1617-24.

42. Smith Gl, Mittendorfer B, Klein S. Metabolically healthy obesity: facts and fantasies. J Clin Invest. 2019;129(10):3978-89.

43. Garcia-Moll X. Obesity and prognosis: time to forget about metabolically healthy obesity. Eur Heart J. 2018;39(5):407-9.

\section{Publisher's Note}

Springer Nature remains neutral with regard to jurisdictional claims in published maps and institutional affiliations.
Ready to submit your research? Choose BMC and benefit from:

- fast, convenient online submission

- thorough peer review by experienced researchers in your field

- rapid publication on acceptance

- support for research data, including large and complex data types

- gold Open Access which fosters wider collaboration and increased citations

- maximum visibility for your research: over $100 \mathrm{M}$ website views per year

At $\mathrm{BMC}$, research is always in progress.

Learn more biomedcentral.com/submission 